\title{
Comprensión del proceso de educación para salud en un programa de atención a la primera infancia, Medellín, Colombia (2014-2015)*
}

\section{Understanding the Health Education Process in an Early Childhood Care Program, Medellín, Colombia (2014-2015)}

\section{Compreensão do processo de educação para saúde em um programa de atendimento a primeira infância, Medellín, Colômbia (2014-2015)}

Fecha de recepción: 24-01-2017 Fecha de aceptación: 20-04-2017 Disponible en línea: 30-05-2017 doi:10.11144/Javeriana.rgps16-33.cpes

Cómo citar este artículo:

Gallego-Osorio GY, Peñaranda-Correa F, Molina-Berrío DP. Comprensión del proceso de educación para salud en un programa de atención a la primera infancia, Medellín, Colombia (2014-2015). Rev Gerenc Polít Salud. 2017; 16 (33): 102-115. https://doi.org/10.11144/Javeriana.rgps16-33.cpes

Gloria Yaneth Gallego-Osorio**
Fernando Peñaranda-Correa ${ }^{* * *}$
Diana Patricia Molina-Berrío****

* Artículo de investigación.

** Autora principal, magíster en Salud Pública; nutricionista dietista, participante del Seminario de Línea de Educación para la Salud, Facultad Nacional de Salud Pública de la Universidad de Antioquia; profesora de la Escuela de Nutrición y Dietética y de la Facultad Nacional de Salud Pública de la Universidad de Antioquia. Correspondencia: Facultad Nacional de Salud Pública, Oficina de Posgrados. Medellín, Colombia: Calle 62 No.52-59, oficina 412. Correo electrónico: glorigalle@gmail.com

*** Doctor en Ciencias Sociales, Niñez y Juventud; magíster en Educación y Desarrollo Humano; magíster en Salud Pública; profesor e investigador de la Facultad Nacional de Salud Pública de la Universidad de Antioquia. Correo electrónico: fernando.penaranda@udea.edu.com

**** Magíster en Salud Pública; profesora e investigadora de la Facultad Nacional de Salud Pública de la Universidad de Antioquia. Correo electrónico: dpatricia.molina@hotmail.com 


\section{Resumen}

Se presenta la comprensión del proceso educativo del programa Buen Comienzo, modalidad de gestación y primer año, como insumo para ampliar los aprendizajes sobre la educación para la salud en general. Esto forma parte de los resultados de una investigación cualitativa, bajo enfoque hermenéutico y orientación etnográfica. El trabajo de campo se realizó mediante observación participante, entrevistas semiestructuradas y revisión de documentos institucionales. Los resultados mostraron que, si bien el programa busca privilegiar el intercambio de experiencias y reconocer los saberes y percepciones de las familias respecto a la crianza —en una relación dialógica con los saberes disciplinares de los agentes educativos-, las prácticas educativas privilegian y legitiman el saber científico biomédico sobre el saber de los adultos significativos. Ello evidenció su peso cultural concretado en procesos educativos fuertemente clasificados y enmarcados según la propuesta de Bernstein, bajo la cual se realizó el análisis del estudio.

Palabras clave: educación en salud; antropología cultural; niñez; política social; cuidado infantil; investigación cualitativa

\section{Abstract}

This article presents the understanding of the educational process of the 'Buen Comienzo' program, in the gestation and first year modality, as an input to expand upon the learning about education for health in general. This is part of the results of a qualitative research, under a hermeneutical approach and an ethnographic orientation. The field work was carried out through participant observation, semi-structured interviews, and the review of institutional documents. Results showed that, although the program seeks to privilege the exchange of experiences and recognize the knowledge and perceptions of families regarding the upbringing -in a dialogical relationship with the disciplinary knowledge of educational agents- educational practices privilege and legitimize scientific biomedical knowledge over the knowledge of significant adults. This came to show its cultural weight, materialized in strongly classified educational processes which are framed according to the proposal of Bernstein, which guided the analysis of the study.

Keywords: health education; cultural anthropology; childhood; social policy; child care; qualitative research

\section{Resumo}

Apresenta-se a compreensão do processo educativo do programa Buen Comienzo, modalidade de gestação e primeiro ano, como insumo para ampliar os aprendizados sobre o ensino para a saúde em geral. Isso forma parte dos resultados de uma pesquisa qualitativa, sob o enfoque hermenêutico e orientação etnográfica. O trabalho de campo realizou-se mediante observação participante, entrevistas semiestructuradas e revisão de documentos institucionais. Os resultados mostraram que, bem que o programa visa privilegiar a troca de experiências e reconhecer os saberes e percepções das famílias respeito da criação — em uma relação dialógica com os saberes disciplinares dos agentes educativos - , as práticas educativas privilegiam e legitimam o saber científico biomédico sobre o saber dos adultos significativos. Isso evidenciou seu peso cultural concretado em processos educativos fortemente classificados e enquadrados de acordo à proposta de Bernstein, sob a qual realizou-se a análise do estudo.

Palavras chave: educação em saúde; antropologia cultural; infância; política social; cuidado infantil; pesquisa qualitativa 


\section{Introducción}

Aunque la educación para la salud se plantea como una dimensión importante de la salud pública, esta no ha tenido el desarrollo esperado (1). Algunos autores (2) consideran que esta práctica es "poco comprendida y no se tiene claridad sobre sus resultados". Al respecto, Serrano, Tones y Whitehead (3-5) argumentan que la educación que imparten los profesionales de la salud se basa en la transmisión e imposición de temáticas a través de posturas tradicionalistas y conductitas, que, además, solo se enfocan en la enfermedad (6).

Algunos autores (3,7-9) han identificado diferentes enfoques en las prácticas de educación en el ámbito de la salud. En primera instancia, están quienes se orientan a la transferencia de contenidos temáticos a través de estrategias informativas. Este tipo de prácticas han sido catalogadas como propias de un modelo biomédico de enfoque positivista que influye de manera decisiva en la forma de pensar y actuar de los profesionales. Los convierte en poseedores de un saber científico que no puede ser discutido y marca distancia con el educando, la cual queda mediada por relaciones de poder que ponen en desventaja a este último, quien termina viéndose como un sujeto carente de conocimientos, incapaz de transformar su realidad $(2,3,9)$.

Por otro lado, se han planteado modelos alternativos que, según sus autores, pretenden ir más allá de la función del modelo biomédico de la transmisión de información y reconocer al educando como poseedor de un saber propio, lo que hace aportes importantes para la construcción conjunta del conocimiento y supone la obtención de mejores resultados en el proceso de formación, en comparación con las prácticas informativas $(2,3,9,10)$. mencionada sobre la educación se refiere al sector salud, una alternativa consistiría en buscar aprendizajes de las experiencias de programas de otros sectores en los cuales la educación tiene un peso destacado. Este es el caso del programa Buen Comienzo.

Buen Comienzo (BC) es un programa dirigido a la atención de la primera infancia creado por el Concejo de Medellín en el 2004. Con posterioridad a la creación del programa, se emite en el 2011 la Política Pública de Atención a la Primera Infancia, mediante el Acuerdo Municipal 058 del Concejo de Medellín, Colombia. De esta manera, el programa se convierte en la forma de materializar la política pública de atención a la primera infancia en la ciudad, al articular los sectores de salud, educación, recreación y cuidado. Su objetivo es promover el desarrollo integral, diverso e incluyente de los niños desde la gestación hasta los cinco años, desde una perspectiva interdisciplinaria del ciclo vital, la protección de los derechos y la articulación interinstitucional (11). Este programa viene atendiendo a 83000 niños, con un presupuesto aproximado de setenta millones de dólares anuales (12).

Una de sus modalidades, Gestación y Primer Año - antes denominada Buen Comienzo Había Una Vez, se creó en el 2009 con el fin de favorecer el desarrollo de los niños desde la gestación: atiende a las madres gestantes, los lactantes y los niños en su primer año de vida. La atención consiste en la intervención educativa por parte de un equipo interdisciplinario compuesto por las áreas de nutrición, psicología, pedagogía infantil y educación física, con el objetivo de fomentar la estimulación cognitiva y motriz, brindar apoyo psicosocial a los niños y sus familias y promover su adecuado crecimiento y desarrollo (11). Igualmente, se entrega un complemento alimentario y se monitorea el estado nutricional mediante la toma de medidas antropométricas (peso y ta1la), tanto del bebé como de la madre gestante y lactante $(11,12)$. 
Este programa entiende la educación como un eje transversal de la atención integral, por lo cual establece una propuesta educativa con una sustentación pedagógica concreta que, por lo demás, se propone como alternativa (11). Comprender la forma como se plantea y se implementa esta propuesta educativa es importante para avanzar en la comprensión de la educación para la salud en general. Para lograr dicha comprensión deben analizarse no solo los documentos institucionales, sino también las prácticas educativas y los significados que los actores atribuyen al proceso educativo.

De acuerdo con todo lo anterior, en el 2014 se inició una investigación de la cual parte el presente artículo y cuyo objeto consistía en comprender el proceso educativo del programa Buen Comienzo en su modalidad de gestación y primer año, del2014 al 2015, con el propósito de enriquecer la teoría y la práctica del programa, contribuir con elementos teóricos que permitan una mayor comprensión de los resultados del programa y avanzar en la comprensión de la educación para la salud en general.

La aproximación teórica para realizar dicho análisis se abordó desde el modelo teórico propuesto por Peñaranda (2), basado a su vez en la sociología educativa desde los aportes de Bourdieu y Bernstein y Passeron $(13,14)$, quienes ven la educación como un medio de reproducción cultural que se da mediante relaciones entre un primer nivel, el micronivel -que corresponde a aquello que acontece en el seno del programa-y un segundo nivel, el macronivel, correspondiente a las instancias sociales, culturales e institucionales que operan como fuerzas externas sobre el micronivel (14).

\section{Materiales y método}

Se realizó un estudio cualitativo desde una perspectiva hermenéutica, por lo cual se toma la interpretación como un proceso subjetivo y, por lo tanto, como una forma de ver la realidad en diálogo con el otro $(15,16)$. Siguiendo una orientación etnográfica $(15,16)$, se buscó comprender cuáles eran los referentes y valores culturales que afectan a aquello que se enseña en el programa (la crianza) y aquello que se considera medio para lograr sus resultados (las prácticas pedagógicas del proceso educativo).

El estudio se basó en comprender la cultura de los actores para interpretar sus actuaciones, siguiendo la propuesta de Geertz (17) para quien el hombre se encuentra inmerso en una trama de significaciones que él mismo ha construido, y este entramado es la cultura.

Entre septiembre del 2014 y abril del 2015 se realizó un estudio de orientación etnográfica caracterizado por la observación participante en el medio donde se realiza normalmente el programa, la realización de entrevistas semiestructuradas a los actores (agentes educativos y adultos significativos) y la revisión de documentos institucionales.

Se seleccionaron dos centros de atención: Manrique y Santo Domingo, en los cuales, a su vez, se escogieron tres familias beneficiarias en cada uno de ellos, de acuerdo con los criterios de selección previamente definidos. El criterio de selección más importante, tanto para los centros como para las familias, establecía que fuera una experiencia considerada exitosa según el personal coordinador de la estrategia y los mismos actores del proceso, como lo plantea Stake (18).

Se asistió a quince encuentros educativos, así como a cinco encuentros educativos en casa; es decir, visitas domiciliarias en compañía de los profesionales. Se realizaron once entrevistas individuales semiestructuradas a diferentes personas beneficiarias del programa (madres gestantes y lactantes y un padre 
de familia) y a cinco profesionales de las diferentes áreas. Asimismo, se hicieron dos entrevistas colectivas al grupo de profesionales para efectuar un nivel de análisis secundario, consistente en profundizar en las categorías preliminares obtenidas.

De forma posterior a los avales institucionales y del comité de ética, se diligenció el consentimiento informado y se realizó el trabajo de campo, cuyas actividades se registraron en un diario de campo. Las entrevistas se grabaron y se transcribieron. Igualmente se obtuvieron grabaciones de las sesiones educativas también transcritas posteriormente.

El análisis etnográfico consistió en clasificar exhaustivamente la información, lo cual generó una cantidad extensiva de códigos que permitieran explorar toda la riqueza de los datos. Dichos códigos fueron agrupados luego en tres grandes categorías planeadas desde el inicio del estudio (la crianza, el proceso educativo y los resultados del programa). Posteriormente, dentro de cada una de estas categorías se conformaron subcategorías descriptivas (19). También se emplearon mapas para organizar mejor la información. Las subcategorías se conformaron de acuerdo con las temáticas tenidas en cuenta en la entrevista semiestructurada y según temas emergentes que surgieron en el proceso de análisis (19).

Este artículo se centra en mostrar la comprensión lograda sobre cómo funciona y cómo se presenta el proceso educativo, cuáles y cómo son las prácticas pedagógicas.

\section{Resultados}

$\mathrm{BC}$ está regido por disposiciones legales que le dan sustento y orientan su quehacer. Dentro 106 de los lineamientos se encuentran el Plan de Atención Integral a la Primera Infancia (PAI), el cual plantea la ruta de acción para garantizar la coherencia con la Política Pública de Atención a la Primera Infancia de la Ciudad de Medellín (11).

El programa concibe la educación como eje transversal a la atención integral; por lo tanto, ha diseñado una propuesta educativa alternativa que guía las prácticas educativas de los profesionales, basada en los lineamientos educativos para el desarrollo integral de la primera infancia, en el cual se encuentran los fundamentos teóricos y metodológicos del proceso educativo, con base en referentes nacionales e internacionales sobre educación inicial (11).

En este sentido, se propone la ejecución de encuentros educativos basados en unos momentos y el uso de los lenguajes expresivos como la ruta metodológica que respalda la educación en el programa (11). Estos encuentros se conciben como experiencias de aprendizaje significativas, basadas en las necesidades e intereses del niño y de la familia, planeadas de acuerdo con el contexto sociocultural y en las cuales se da importancia a la participación activa de las familias y los niños como actores principales del proceso, con el fin de posibilitar el intercambio de experiencias y vivencias frente a la crianza y la educación de los niños.

Asimismo, se propone emplear las expresiones artísticas o lenguajes expresivos como una forma de desarrollar una propuesta educativa diferente, lo que posibilita la aproximación al sujeto desde las dimensiones cultural, sensitiva y corporal como una manera de expresar sentimientos y emociones, además de tener otras formas de relacionarse con el entorno. Por otro lado, se habla de la pedagogía crítica como uno de los sustentos pedagógicos del programa. Pese a lo anterior, se encontró cierto contrasentido al contrastar la teoría y la práctica del programa. 
A continuación, se presentan las impresiones de los agentes educativos (AE) en relación con el proceso educativo llevado a cabo. En primera instancia, se describen los propósitos percibidos, después la orientación, seguidamente algunos elementos relacionados con la planeación de sesiones educativas, para continuar con las principales características de la metodología realizada y finalizar con los resultados observados.

$\mathrm{Al}$ preguntarle a los $\mathrm{AE}$ sobre los propósitos del proceso educativo, ellos argumentan que los encuentros se conciben como medio para transformar las experiencias, los imaginarios y las percepciones en la familia que se consideren barreras sociales y culturales respecto a una crianza concebida como adecuada por el AE, aunque, por otro lado, se habla de reconocer y fortalecer la dimensión cultural del niño y de su familia. En este orden de ideas, el $\mathrm{AE}$ refiere que respeta y tiene en cuenta los conocimientos de los adultos significativos (AS), pero siente la necesidad de hacer que estos cambien de mentalidad:

\section{[...] es cambiar un poquito el chip de las fami- lias, de entender muy bien la responsabilidad de ser padre y de ser madre, de generar mucha educación, [...] cambiar mentes a través de la educación.}

No obstante, desde los lineamientos educativos se define como propósito desarrollar competencias en los AS, para que estos se empoderen y se apropien de su proceso de crianza y sean los principales educadores de los niños, como una manera de garantizar las condiciones para lograr el desarrollo integral de los niños y las niñas:

Yo pienso que uno de los propósitos fundamentales que nosotros procuramos plantearles a las familias es empoderarlas; o sea, que se apropien de su rol como educador o educadora de ese bebé que ya viene en camino o que ya está en brazos; empoderarlos en un montón de herramientas, en educar, en orientar; desarrollar potencialidades en esas familias”.

Los profesionales destacaron como eje central del programa la educación y la estimulación adecuada. Así, mediante la práctica educativa, el AE hace uso de su conocimiento y de su repertorio de actividades (demostraciones) para que la crianza esté centrada en la estimulación adecuada:

\section{[...] y que ese acompañamiento tenga siempre un objetivo y un propósito que no es sentarme a darle la sopa al bebé, a embutírsela [...] el programa te enseña, mamá, cómo sentarte con tu bebé frente a frente a darle esa compota [...] sentarme a hacer una actividad motriz con mi bebé.}

El AE recalca la importancia de la educación en el programa, con lo cual manifiesta su compromiso con la dimensión pedagógica:

Texto de la sesión:

AE [pedagoga]: "Buenos días, familias [... las felicito porque sacar un rato de tiempo en el día, con todas las cositas que hay que hacer en la casa, no es fácil; sobre todo, ser conscientes de que cuando venimos acá estamos dando muestra de amor, calidad de vida, compromiso, ¿cierto? [...] que no venimos acá solo por el complemento”.

En relación con la planeación de los encuentros educativos, se hace referencia a la importancia de leer el contexto y caracterizar las familias mediante la observación constante durante los encuentros y las visitas, para identificar sus necesidades e intereses, que se concretan en detectar lo que para los profesionales son falencias o desconocimientos del AS:

[...] la planeación; por eso digo que la lectura es muy sutil porque ni siquiera es preguntarles: ¿familias, qué temas les gustarían? Es 
como observar las necesidades; por ejemplo: estas mamás están hablándoles muy duro a los niños, y si vemos que la situación se repite, entonces al próximo mes hablaremos de norma.

Luego de identificar las necesidades, esto es, los problemas detectados en las familias, el AE debe tener presente otro aspecto de la planeación: pensar el encuentro más allá de las necesidades del adulto, para que pueda tenerse presente al niño como actor central.

En cuanto a las características de la metodología, según los profesionales, una muy importante es la construcción de relaciones horizontales con las familias, donde se respetan sus puntos de vista y se les admite como capaces de criar:

\begin{abstract}
Si tienen dificultades [...] nosotros tratamos de solucionarlas, pero siempre que sea primero la familia la que se responda porque validamos sus conocimientos [...] claro que siempre hay que reforzarles, pues ellos tienen derecho a aprender.
\end{abstract}

Lo anterior implica que el agente educativo es quien define los comportamientos que considera deben ser adoptados por los AS:

\section{[...] que el agua de cilantro para que duerma, etc.; entonces aclaramos: "sí, mamá, eso es lo que dicen [otras personas], pero lo que dice la Organización Mundial de la Salud — lo que dicen los nutricionistas- es esto.}

El AE es quién tiene los conocimientos para mostrarle a la familia cómo garantizar que el niño logre su desarrollo, qué actividades deben cumplir para estimularlo:

[...] también tratamos de mostrarle a las familias lo vivencial y el cómo pueden garantizar esos desarrollos; entonces siempre que se realiza una actividad central hay unas devoluciones donde también se les cuenta a las familias para qué se está haciendo [...].
De acuerdo con las orientaciones teóricas del programa, los agentes educativos conciben los encuentros como espacios lúdicos y formativos en los cuales las familias y los niños interactúan, aprenden e intercambian saberes y experiencias sobre la crianza. Para ellos, esta se basa en la utilización de diferentes recursos didácticos o lenguajes expresivos, y no en la utilización de técnicas consideradas por ellos conductistas y "transmisionistas", tales como las charlas. En este sentido, destacan que BC posee una metodología que guía el quehacer del profesional:

La metodología con la que trabajamos tiene que ser todo el tiempo de interacción [...] diferente a otras estrategias metodológicas.

\section{[...] entonces no es directamente llevar una cátedra porque nuestra metodología no se maneja desde allí, de ir a dar un taller reflexivo o vivencial, sino desde los encuentros edu- cativos a través de los lenguajes expresivos.}

Además, los $\mathrm{AE}$ tienen la convicción de que los lenguajes expresivos constituyen una vía para crear o fortalecer el vínculo afectivo con el niño, la mamá y la familia. Dicha metodología es coherente con la orientación de que la educación tiene como destinatario final el niño o la niña, por lo cual, entonces, los AS adquieren una connotación instrumental:

[...] otro sentido del juego es que genera aprendizaje, pero a través del juego también se fortalece el vínculo afectivo entre la mamá y todo el núcleo familiar, y esa es la base.

\section{[...] la intención central es que las familias no lleguen a una charla. [...] La charla es para la mamá, mas no para el niño.}

Si bien existe consenso respecto a la idea de que los encuentros no son charlas ni se hacen de manera magistral, se dan diferentes concepciones acerca de la metodología, pues 
mientras unos profesionales consideran que esta no se refiere a la ejecución de talleres reflexivos o vivenciales, otros la describen como una metodología vivencial y experiencial según la cual las personas sienten y vivencian las actividades, además de que se trasciende el hecho de contar, de dar información, para abrir posibilidades a las familias de interiorizar aprendizajes, de irse con más conocimientos:

[...] es muy experiencial; o sea, nosotros les proponemos unas actividades y la idea es que las madres las vivan y las desarrollen porque no es simplemente contar y contar, sino que ellas estén desarrollando la actividad durante el encuentro educativo porque hemos descubierto que a través de esa metodología experiencial se genera mayor interiorización del aprendizaje, entonces la familia se va con más conocimientos.

Uno de los asuntos que se consideran claves para lograr que las familias se comprometan con el proceso educativo es el establecimiento de acuerdos para el desarrollo de las sesiones educativas, acuerdos que son planteados por los AE como una construcción colectiva, aunque estos se encuentran trazados desde los lineamientos del programa:

Texto de la sesión:

$\mathrm{AE}$ [educador físico]: Vamos entonces a recordar los acuerdos que entre todos construimos el día que ingresaron; o sea, en febrero; ¿se acuerdan? ¿Cuáles fueron los acuerdos?

\section{AS: Puntualidad $[\ldots]$}

AS: Escuchar a los profesores y escuchar a los compañeros.

$\mathrm{AE}$ [educador físico]: Muy bien, pero que esa escucha sea activa, participativa, respetuosa y no solamente porque hable mi compañera o porque hable yo. Cualquiera que quiera hablar, se le va a poner toda la atención [...] Ninguna pregunta carece de importancia; todas requieren la atención necesaria [...].

$\mathrm{AE}$ [educador físico]: También muy buena disposición $[\ldots]$; entonces vienen a las actividades, pero a participar y, lógicamente, a interactuar con el niño y con la niña.

Adicionalmente, las actividades de las sesiones deben replicarse en el hogar, lo cual debe verificarse como una forma de evaluar los resultados y evidenciar los avances en el desarrollo del niño. Por lo tanto, también se establecen compromisos para aplicar los aprendizajes logrados en la sesión a las prácticas de crianza en los hogares de los AS:

Nosotros les decimos que lo importante es que lo que hagamos acá lo sigan practicando porque, si no, no nos garantizan que haya avances en el bebé. El compromiso de ustedes es que mes a mes lo que trabajamos lo pongan en práctica en casa; entonces, es una de las formas que llamo tareas para las familias.

Las mamás nos cuentan avances si recuerdan algo del encuentro anterior y lo han practicado en casa.

Se encuentra una situación paradójica, pues por un lado el $\mathrm{AE}$ plantea que las familias son las que deciden si acogen o no sus recomendaciones, pero durante las sesiones utilizan expresiones como tienes que hacer, tienes que evitar, puede comer de todo.

\section{[...] nosotros les decimos qué es lo adecuado, pero son ustedes, las mamás, quienes deciden si toman o no la recomendación.}

Otra característica tiene que ver con el trabajo simultáneo de varios temas, que pueden reforzarse mutuamente por medio del desarrollo de una actividad que los integre: 
[...] en esta actividad conjunta vamos a hacer una actividad con respecto al tema; por ejemplo, vamos a rasgar papel globo [...] para pasarlo por el cuerpo del bebé; y cuando hago esto, voy a pensar en la fruta o el alimento que más le gusta a mi hijo. Ejemplo: le gusta mucho el banano, ¿y el banano con qué parte del cuerpo rima, mamá? iAy!, con las manos, entonces vamos a hacer una canción con las manos.

Otra estrategia utilizada para estimular la participación de los AS es darle la posibilidad a las familias de que ellas mismas infieran el tema y luego explicarlo desde la teoría, para saber cuál era la actividad, para qué les sirve y cómo la pueden aplicar:

\section{[...] antes de decirles el tema, les permitimos que los deduzcan y, luego, después de escu- char el aporte de ellas, se hace la devolución de la temática contándoles de manera técnica qué estaban haciendo [...], cómo lo pueden aplicar en casa.}

Supongamos que estamos hablando de sonidos onomatopéyicos [...] y la mamá tiene derecho a saber que eso se llama sonido onomatopéyico, que sirve para estimular a su bebe $[\ldots]$, entonces, en ese momento, se les cuenta la parte técnica.

Como resultado final, el $\mathrm{AE}$ manifiesta que las familias logran acomodarse a la estructura del programa y responden positivamente a lo que se les propone desde los encuentros; en tanto se vuelven comprometidos, acceden a participar de las actividades, venciendo la pena y la resistencia por tener que hacer actividades a las que no están acostumbrados, como cantar o hablarle al bebé desde el vientre. De esta manera, atienden las demandas del programa, asisten a las sesiones movidos por los aprendizajes, y no por la ayuda alimentaria que se ofrece, y adoptan el lenguaje técnico de los profesionales.
Así, el AE plantea que reconoce fortalezas en el AS, ya que considera que adaptarse al proceso educativo significa volverse más comprometido, empoderarse, entender su papel en la crianza como principal educador de sus hijos y saber hacia dónde lo quiere llevar el programa. No obstante los resultados expresados por los profesionales, también se vuelven un motivo de frustración para ellos ver que las familias no cumplen las recomendaciones que se les hacen, o hacen lo contrario de lo que lo que se les enseña. Esta es otra de las paradojas que se observan en el proceso educativo descrito y que a continuación se pretende analizar.

\section{Discusión}

El programa BC, a diferencia de los programas del sector salud que tienen que ver con la crianza, toma esta de manera explícita como su eje central y despliega una propuesta pedagógica y didáctica concreta fundada en la educación inicial y en los lenguajes expresivos como medio para lograr una relación dialógica con las familias y promover la estimulación adecuada. Además, se plantea un ideal pedagógico orientado desde la pedagogía crítica (11).

La propuesta pedagógica se lleva a la práctica a través de equipos con profesionales de diferentes disciplinas, incluidos profesionales con formación pedagógica. Los educandos manifiestan una clara aceptación y valoración de las prácticas pedagógicas, pues encuentran un espacio en el que resuelven las angustias e inquietudes cotidianas sobre la crianza y la gestación en el marco de relaciones cercanas y estimulantes, algo que también contrasta con los procesos educativos de los programas del sector salud $(20,21)$.

Los resultados de la educación son reconocidos por los AS, quienes manifiestan los aportes de la educación para apoyar la crianza que 
llevan a cabo. A su vez, el programa ha recibido reconocimientos nacionales e internacionales por su orientación intersectorial, puesto que ha logrado vincular diferentes sectores públicos y privados en pro de la atención integral a la primera infancia, así como por sus principios pedagógicos y por los resultados sobre las familias con las cuales trabaja (12).

A pesar de las grandes fortalezas que exhibe el programa para realizar un proceso educativo más pertinente -mediante los hallazgos de este estudio referentes a la concepción que se tiene de la crianza y a la forma como se ejercen las prácticas educativas-, se identifican paradojas pedagógicas que parecen entrar en contradicción con la pedagogía crítica y la propuesta dialógica que se pretende realizar desde el programa.

Esto se debe a que, aun cuando se argumenta una educación basada en relaciones horizontales con las madres, a quienes se visualiza como sujetos activos con un conocimiento valioso, capaces de criar, este sustento teórico, por momentos, choca con la concepción de un adulto que debe ser guiado, que requiere capacitarse para aplicar el modelo de crianza adecuado como un proceso basado en la estimulación adecuada y que estaría más cercana a la idea de educación bancaria de Freire (22).

Así, las prácticas pedagógicas se orientan a desarrollar competencias en las familias y a empoderarlas en su rol como educadores, con el fin de que esto redunde en el bienestar de los niños y garantice su desarrollo integral, lo que termina configurando una tendencia a ver el adulto con una función más instrumental para lograr los propósitos del programa. Dicha centralidad en el niño es coherente con la fundamentación pedagógica cimentada en los preceptos de la educación inicial y el desarrollo integral del niño. Esto puede llevar a desconocer la crianza como un proceso ontológico, sociocultural e histórico que compromete al adulto como ser individual y social quien tiene sus propias experiencias y concepciones sobre lo que significa la crianza (23).

De esta manera, los lineamientos educativos del programa demarcan los objetivos y la ruta que seguir por parte de los profesionales para llegar a unas metas establecidas, las cuales requieren un proceso educativo orientado hacia el logro de comportamientos que permitan la estimulación adecuada y el desarrollo integral de los niños. Los agentes educativos son los llamados a divulgar una concepción de crianza que se ha construido con la influencia del programa y desde la postura de ellos como profesionales; es decir, enseñar el deber ser de la crianza. Bernstein (14) habla de una educación centrada en la performance, la cual considera conservadora y que implica un proceso de reproducción cultural que Bourdieu (13) relaciona con la educación tradicional.

En el campo de la salud, estas prácticas educativas corresponden a lo que algunos autores $(3,7)$ denominan educación para la salud tradicional, al centrarse en la transmisión del conocimiento científico y valores culturales particulares que "determinarían" una crianza adecuada, la cual está cruzada por connotaciones morales (lo bueno y lo malo en la crianza). Los profesionales consideran que deben transmitir conceptos teóricos (científico-disciplinares) a la familia, justificando que es un derecho de estas, pero también basados en una sustentación moral de que todo aquello es por el bien del bebé; esto evidencia cómo el discurso pedagógico es una combinación de contenidos científico-disciplinares y culturales o regulativos $(14,23)$.

$\mathrm{Al}$ igual que en otros programas similares en el campo de la salud (1-3), las prácticas pedagógicas se orientan a obtener comportamientos deseados según lo definido por el programa desde sus lineamientos educativos, 
para lo cual se emplean acciones de estímulo y respuesta y de reforzamiento que consisten en formulación de preguntas y en demostraciones de actividades que el AS debe repetir; asimismo, en el establecimiento de tareas que deben cumplirse por fuera del encuentro y en la verificación de su realización en la siguiente sesión: así se evalúan los resultados. Estos rasgos corresponden a prácticas educativas que Serrano (3) identifica como persuasivo motivacional y Not (24) relaciona con métodos pedagógicos herteroestructurantes, porque el proceso formativo depende de otro. Aquí la centralidad estaría en el educador y no en el educando, como sería el caso de los métodos interestructurantes (las propuestas constructivistas).

De esta manera, se hace evidente un aparente contrasentido entre la propuesta pedagógica del programa y las prácticas educativas, lo cual concuerda con hallazgos de estudios similares que plantean que los profesionales en salud terminan ejerciendo prácticas educativas tradicionales en programas que buscan llevar a cabo una educación alternativa (2-4). ¿Pero cómo sucede esta paradoja, de la cual los actores pueden no ser conscientes? E1 análisis sociológico que puede hacerse con el concepto de medicalización (25) y desde los trabajos de Bernstein (14) y de Bourdieu y Passeron (13) constituyen un aporte valioso para comprender este fenómeno.

De acuerdo con Bourdieu y Passeron y Bernstein, estas posiciones paradójicas en la educación pueden comprenderse si se consideran las fuerzas sociales que actúan desde fuera del escenario educativo propiamente dicho. En este caso tienen que ver con la influencia del conocimiento científico, impulsado especialmente por el campo de la medicina y la biología y que se ha materializado en el modelo biomédico. Y resulta más característico de los profesionales del área de la salud.
En el modelo biomédico, a la par con la ciencia positivista, el agente de salud como poseedor de un saber disciplinar establece parámetros para distinguir la salud de la enfermedad, esto es, lo que es normal y lo patológico, pero también lo que es adecuado y lo inadecuado, aspecto que le otorga una posición de poder en la sociedad y le da la potestad de emitir juicios $(25,26)$. En este sentido, se dan en la práctica relaciones mediadas por el profesional experto y el paciente (que no tiene el conocimiento experto, pues solo posee el conocimiento práctico o su experiencia) $(26,27)$. Tiene lugar, entonces, un proceso de medicalización, en el cual se da la imposición del punto de vista del experto, en el control, la administración y la planificación de la vida, lo que incluye el empleo de lenguaje médico para la descripción de lo cotidiano, en este caso en lo referente a la crianza (24).

Por otro lado, desde la perspectiva de la sociología educativa, lo anterior se explica porque existen fuerzas externas o de "clasificación" (14) que se refieren a todos aquellos asuntos de orden social — como las políticas en primera infancia y en salud- que responden a discursos científicos y culturales particulares al definir aquello que se considera verdad, correcto, adecuado o bueno. Con este tipo de lineamientos se definen los valores y los contenidos para los programas educativos. Dichos valores y formas de comprender el mundo son los que priman a la hora de determinar los significados legítimos en el escenario educativo y que pueden establecer una distancia entre los valores de quienes elaboran y reproducen dicho discurso (funcionarios y agentes educativos que construyen las políticas y operan los programas) y quienes lo reciben; es decir: los educandos, quienes por lo general provienen de clases sociales diferentes (14).

Estas diferencias de clases se manifiestan también en el ámbito de las prácticas educativas; así, una diferencia de habitus entre 
el AE y el AS, debido a su origen, significa que ambos tienen diferentes formas de ver el mundo y de desenvolverse en él, como producto de su historia y de la clase social a la que pertenecen (13).

Por un lado, los adultos significativos provienen de clases populares y poseen códigos de lenguaje restringidos (14); esto no implica tener menos nivel cognitivo, sino poseer una retórica del lenguaje más concreta. $\mathrm{El} \mathrm{AE}$, por su parte, tiene un habitus diferente, como producto de su clase social y de su paso por la educación profesional (13), lo que implica posesión de códigos de lenguaje elaborados (14); es decir, que se comunica utilizando un lenguaje más complejo o abstracto, asunto de gran relevancia para él, pues se convierte en una vía para validar lo que hace y lo que dice: sustentar con conceptos técnicos (científico-disciplinares).

Además de las fuerzas de clasificación, existen fuerzas internas o de enmarcamiento referentes a todos los elementos que configuran la práctica pedagógica propiamente dicha. Siguiendo a Bernstein (14), el modelo educativo que se analiza posee un enmarcamiento fuerte, pues su control se encuentra en las manos del educador, quien termina aplicando una perspectiva de educación tradicional que, para Bourdieu y Passeron (13), constituye la más eficiente para "imponer" un contenido pedagógico (o arbitrio cultural) configurado por significados provenientes del conocimiento científico y de un referente cultural particular.

Entonces, el AE adapta el discurso dado por las políticas, por los lineamientos del programa y por las entidades encargadas de operar el programa influenciado por su propia experiencia personal y cultural, movido por las fuerzas de clasificación y enmarcamiento, lo cual se conoce como el proceso de recontextualización (14). Este discurso recontextualizado o adaptado (desde el que se define lo que es verdadero, correcto y bueno) produce un control sobre el potencial de significados respecto a lo que se pretende enseñar; es decir, que define aquellos significados que son "legítimos" o relevantes, la secuencia de los contenidos y el tiempo o ritmo para transmitirlos (14).

En este sentido, el AE siente la necesidad de validar los significados que se producen en el escenario educativo cuando contrasta su discurso con la visión de crianza de los adultos. Valida aquellos significados que concuerdan con su discurso pedagógico y rechaza aquellos diferentes, nombrándolos como arraigos culturales o creencias.

Según Bourdieu y Passeron y Bernstein $(13,14)$, este proceso se hace de manera inconsciente, es decir, que está "enmascarado", porque los actores no se dan cuenta de estas relaciones de poder, y así el $\mathrm{AE}$ asume una postura moral que lo lleva a transmitir sus conocimientos (que considera los verdaderos y necesarios) para que las familias tengan la información adecuada. Por esto, considera que el AS tiene "derecho a aprender", esto es, que tiene derecho a adquirir el saber verdadero, el saber científico. Además, propicia espacios para que el AS exprese su saber cómo una manera de reconocerlo, pero siente que ese saber tiene que validarse o respaldarse con su discurso (saber disciplinar y cultural), pues este último forma parte de la experiencia cotidiana (es un saber práctico y no científico).

Se evidencia así que en estos programas también se construyen relaciones de poder y se reproducen las desigualdades de clase, retomando las propuestas de Bourdieu, Passeron y Bernstein $(13,14)$ sobre el tema de la educación, no solamente en el medio de la educación formal, sino en otras instancias de educación no formal, como la de este tipo de programas. 


\section{Conclusión}

Se pueden obtener importantes aprendizajes para la salud pública y, más concretamente, para el campo de la educación para la salud, a partir de esta experiencia investigativa. En primer lugar, está la importancia que este programa le otorga a la educación. Es clara una decidida y explícita fundamentación pedagógica que pretende promover prácticas educativas alternativas a una educación tradicional. Esto contrasta fuertemente con la situación del sector salud, en el que muchos programas plantean la importancia de la educación, pero con un desarrollo pedagógico y didáctico que resulta mínimo, lo cual evidencia un contrasentido entre la teoría y la práctica.

Este estudio evidencia, sin embargo, que no es suficiente una buena fundamentación pedagógica al analizar la aparente contradicción entre la teoría y la práctica encontrada. Así, el discurso pedagógico, en algunos casos, se termina legitimando desde la ciencia biomédica, con lo cual se comprende el poder del discurso biomédico y medicalista en la sociedad. Por esta razón, surge entonces la importancia de tener en cuenta las fuerzas sociales a la hora de analizar la educación. Conocer estas fuerzas sirve para avanzar hacia un proceso más coherente entre los deseos teóricos de una educación crítica-alternativa y las prácticas que no logran romper del todo con las amarras de una educación tradicional.

De acuerdo con Freire, este proceso de coherencia requiere una constante reflexión que permita volver a la teoría para regresar a una práctica enriquecida, reconociendo que es un proceso arduo de cambio del educador y de las condiciones socioculturales que condicionan la educación.

Finalmente, es necesario destacar la im114 portancia de centrar, de manera explícita, el programa en la crianza, pues la tradición biomédica del sector salud tiende a soslayar las dimensiones socioculturales e históricas de la crianza y centrarse en las alteraciones del crecimiento y desarrollo de los niños y las niñas como se plantea desde la Resolución 412 del 2000, del Ministerio de Salud. Plantear la crianza como complejo histórico, sociocultural y ontológico fortalece el esfuerzo por promover una perspectiva orientada desde la salud concebida como vida, y no solo desde la prevención de la enfermedad. Se evidencia aquí una forma de superar la "enfermología pública", como lo deseaba Edmundo Granda (28).

\section{Referencias bibliográficas}

1. OMS. Health Education: theoretical concepts, effective strategies and core competencies. Cairo: OMS; 2012.

2. Bastidas M, Acevedo A, Pérez FN, Torres JN. E1 diálogo de saberes como posición humana frente al otro: referente ontológico y pedagógico en la educación para la salud. Invest Educ en Enferm. 2009 mar; 27(1):104-11.

3. Serrano MI. Educación para la salud y participación comunitaria. Madrid: Díaz de Santos; 1990.

4. Tones K. Reveille for radicals. The paramount purpose of health education? Health Educ Res 2002;17(1):1-5.

5. Whitehead D. Health promotion and health education: advancing the concepts. J Adv Nurs. 2004; 47(3):311-20.

6. Greene W, Simons Morton B. Educación para la salud. México: Interamericana- McGraw Hill; 1988.

7. Figueiredo MFS, Rodrigues-Neto JF, Leite MTS. Modelos aplicados às atividades de educação em saúde. Rev Bras Enferm, Brasília 2010 ene-feb; 63(1):117-21.

8. Duarte J, Gallego T, Parra P. Análisis de programas sobre la atención y el desarrollo a la primera infancia. Rev Latinoam Cienc Soc Niñez Juv. 2011;9(2):105-43.

9. Díaz PA. Concepciones teóricas sobre la teoría en educación para la salud: revisión sistemática. Invest Educ Enferm. 2012; 30(3):1-12

10. Salci MA, Maceno P, Rozza SG, Silva DMGV, Boehs AE, Heidemann ITSB. Health education and its theoretical perspectives: a few reflections. Text Context Nursing. 2013 ene-mar; 22(1):224-230.

11. Colombia. Secretaría de Educación de Medellín. Resolución 12760 de 2012 por medio de la cual se regula la prestación del servicio de atención integral a la primera infancia en el desarrollo del programa Buen Comienzo. Medellín: La Secretaría; 2012. 


\section{COMPRENSIÓN DEL PROCESO DE EDUCACIÓN PARA SALUD EN UN PROGRAMA DE ATENCIÓN A LA PRIMERA INFANCIA, MEDELLÍN, ColoMBIA (2014-2015)}

12. Arango C. Diseño e implementación de un sistema de evaluación del programa Buen Comienzo, Medellín 2013-2014 [Tesis de grado para optar al título de doctor en epidemiología]. Medellín: Facultad Nacional de Salud Pública; 2013.

13. Bourdieu P, Passeron JC. La reproducción: elementos para una teoría del sistema de enseñanza. México: Fontamara; 1998.

14. Bernstein B. La estructura del discurso pedagógico. Madrid: Morata; 2001.

15. Ríos T. La hermenéutica reflexiva en la investigación educacional. Revista Enfoques Educacionales. 2005;7(1):51-66.

16. Herrera JD. La compresión de lo social: horizonte hermenéutico de las ciencias sociales, segunda edición. Bogotá: Cinde; 2010.

17. Geertz C. La interpretación de las culturas. Barcelona: Gedisa; 2005.

18. Stake R. Case Studies. En: Denzin N, Lincoln Y, compiladores. Handbook of qualitative research, segunda edición. Thousand Oaks, California: Sage Publications, 2000:235-454.

19. CoffeyA, Atkinson P. Encontrar sentido a los datos cualitativos. Estrategias complementarias de investigación. Medellín: Editorial Universidad de Antioquia; 2003.

20. Muñoz LA, Sánchez X, Arcos E, Vollrath A, Bonatti C. Vivenciando la maternidad en contextos de vulnerabilidad social: un enfoque comprensivo de la fenomenología social. Rev Latino-Am Enfermagem [Internet]. 2013;21(4): [7pantallas] [acceso: $14 \mathrm{de} \mathrm{fe-}$ brero del 2016]. Disponible en: www.eerp.usp.br/rlae 21. Merighi M, Rodrigues R. Domingos S. Care needs of pregnant women with a private health insurance: a comprehensive social phenomenology approach. Rev Latino-Am Enfermagem. 2007 sept-dic; 15(5):914-21.

22. Freire P. Pedagogía del oprimido. México: Siglo XXI; 1975.

23. Santillan L. Escuela, nuevas configuraciones familiares y cambio sociocultural. En: Argentina, Ministerio de Educación, Ciencia y Tecnología de la Nación, Dirección Nacional de Gestión Curricular y Formación Docente Área de Desarrollo Profesional Docente. Cine y Formación Docente 2006. Ciudad de Neuquén; 2006.

24. Not L. La pedagogía del conocimiento. Bogotá: Fondo de Cultura Económica; 2000.

25. Foucault M. Historia de la medicalización. Educ Med Salud. 1977;11(1):3-25.

26. Lolas M. Camino para un eficaz encuentro interpersonal. Rev EE. 1998;1(1):121-26.

27. Berger P, Luckmann T. La construcción social de la realidad. Buenos Aires: Amorrortu; 1968.

28. Granda E. La salud y la vida: Perspectivas de la salud pública para el siglo XXI, volumen 1. Ecuador: OPS; 2009 . 